\title{
Huerto de cítricos plantado con sistema de cero labranza y sistema convencional
}

\section{Citrus orchard planted with no tillage and conventional systems}

\author{
Carmen Silvia Vieira Janeiro Neves ${ }^{1,4^{*}}$; João Tavares Filho ${ }^{1}$; Osmar Rodrigues \\ Brito $^{1}$; Fábio Yamashita ${ }^{2,4}$; Valdomiro Tormem ${ }^{3}$; Inês Cristina de Batista Fonseca ${ }^{1,5}$
}

\section{Resumen}

El sistema de cero labranza ha traído muchas ventajas en la protección del suelo, sin perjudicar el rendimiento de los cultivos anuales. Este sistema ha sido adaptado para los huertos citrícolas, realizándose la plantación en surcos abiertos en terrenos anteriormente ocupados por pastizales. El trabajo tuvo como objetivo evaluar el desarrollo de las plantas y del sistema radicular, producción, características del suelo en un huerto plantado en dos sistemas de labranza. El estudio fue realizado en el Noroeste de Paraná, en un oxisol con textura media, en plantas de naranja 'Valência' con patrón de lima Rangpur. Los tratamientos evaluados fueron: cero labranza (apertura de surcos y plantación) y plantación convencional (arado de discos y grada, seguido de apertura de surcos y plantación). La producción fue de 86 a $129 \mathrm{~kg}$ de frutos por planta, sin diferencia significativa entre los tratamientos. Los sistemas de plantación tampoco afectan el desarrollo de las plantas y de las raíces y la calidad de los frutos. En el sistema de plantación convencional, el suelo presenta mayor resistencia a la penetración en la interlínea, que en el sistema de plantación con cero labranza.

Palabras-clave: Citrus sinensis, Citrus limonia Osbeck, plantación, raíces

\begin{abstract}
No tillage system has brought many advantages to soil protection, without reducing the yield of annual crops. This system has been adapted for Citrus orchards, with the plantation of trees in furrows opened in areas previously occupied by pastures. This work had as objective to evaluate tree development, root system, yield, and soil characteristics in an orchard planted in two systems. The study was made in Parana State, Brazil, in a medium texture oxisol with 'Valência' orange (Citrus sinensis L.) trees with Rangpur lime rootstock (Citrus limonia Osbeck). The treatments were no tillage (furrowing and plantation) and conventional plantation (hole area plowing and disk harrowing before furrowing and plantation). The yield was $86-129 \mathrm{~kg}$ of fruits per plant, without significant difference between treatments. The plantation systems either do not affect the development of the plants, the amount of roots and the fruit quality. In the conventional plantation system the soil has greater resistance to penetration in inter rows than in the zero tillage system plantation.
\end{abstract}

Key words: Citrus sinensis L. Citrus limonia Osbeck, planting, roots

\footnotetext{
${ }^{1}$ Professores Dr. Universidade Estadual de Londrina, UEL, Departamento de Agronomia, C. P. 6001. CEP 86051 990, Londrina, PR.E-mail: csvjneve@uel.br, tavares@uel.br, osmar@uel.br, inescbf@uel.br

2 Prof. Dr. Universidade Estadual de Londrina, UEL, Depto. de Ciência e Tecnologia de Alimentos. C.P. 6001. CEP 86051-990, Londrina. PR. E-mail: fabioy@uel.br

${ }^{3}$ Paraná-Citrus S.A, Paranavaí, PR. E-mail: vtormem@hotmail.com

${ }^{4}$ Bolsista de Produtividade em Pesquisa pelo Conselho Nacional de Desenvolvimento Científico e Tecnológico, CNPq.

${ }^{5}$ Bolsista de Produtividade em Pesquisa da Fundação Araucária

* Autor para correspondência.
} 


\section{Introducción}

En los últimos años el cultivo de cítricos ha adquirido gran importancia económica en la provincia de Paraná, aumentando a cada cosecha el área ocupada. La naranja es la fruta producida en mayor cantidad en la provincia, principalmente en la región noroeste, donde ha sido implantada en las áreas previamente ocupadas por pastos. Los suelos de esta región, geológicamente originarios del arenito Caiuá, poseen pocas reservas, tanto de minerales, cuanto de sustancia orgánica, presentando alta susceptibilidad a la erosión (FIDALSKI, 1997).

El sistema de la plantación directa, que en las culturas anuales ha traído ventajas a la conservación de los suelos (MUZILLI, 1999), fue extendido para las culturas perennes, y se ha utilizado por los productores de cítricos del noroeste del Paraná, que hacen la preparación para la plantación en rayas de 1,5-2,0 de ancho que coinciden con las líneas de la plantación, en las tierras ocupadas anteriormente por pastos o hacen surcos directamente en los pastizales (RUFINO; MUZILLI; PAVAN, 1992). Pero, el efecto de este sistema en el funcionamiento de las plantas cítricas, así como en el suelo de los huertos, aún no fue estudiado.

El conocimiento de la tasa de crecimiento, de extensión y distribución del sistema radicular es esencial para la citricultura por su importancia en la absorción del agua y de los nutrientes, de almacenaje de las sustancias de la reserva y síntesis hormonas. Por otra parte, las raíces son afectadas directamente por el manejo del suelo, fertilizaciones e irrigación. Trabajos han demostrado, en huertos cítricos ya formados, que la ausencia de labranza de la tierra en el espacio entre las hileras de plantío, es la mejor opción para la conservación de las cualidades físicas del suelo (CARVALHO et al., 2002). Pero hay poca información sobre este tema para la fase de implantación de los huertos (AULER et al., 2004; 2008). La búsqueda para los sistemas de plantación de cítricos convenientes a las condiciones locales ajustadas y más a la conservación de la tierra, también se ha acentuado para otros investigadores, como Martins y Pinto (2004).

El actual trabajo tuvo como objetivo evaluar el desarrollo y la producción de las plantas, la calidad de las frutas, la extensión y la distribución del sistema radicular, y analizar las alteraciones de las características físicas y químicas de un Latossolo rojo distrófico de textura media, en un huerto cítrico implantado con el sistema convencional y en sistema de plantación directa en rayas en el noroeste del Paraná

\section{Materiales y Métodos}

El estudio se realizó en un huerto comercial en la granja San José, en Planaltina del Paraná, en la región noroeste de la provincia. El clima, según la clasificación de Koppen, es del tipo del Cfa, húmedo subtropical sin estación seca, con un verano caliente (temperatura media del mes más cálido $>22^{\circ} \mathrm{C}$ ) con tendencia a la concentración de las lluvias e invierno con heladas poco frecuentes (temperatura media del mes más frío $<18^{\circ} \mathrm{C}$ ). El suelo de la región es clasificado como Latossolo Rojo distrófico, con textura media.

El huerto fue implantado en 1991, con naranja 'Valencia' (Citrus sinensis L.) en patrón de lima Rangpur (C. limonia Osbeck), con marco de plantación de 7,5 x 4,0 m. Los dos tratamientos de preparación del suelo fueron: cero labranza con la plantación directa en rayas (apertura de surcos y plantación) y plantación convencional (aradura, grada de discos, apertura de surcos y plantación). Después de la implantación, el área del huerto era manejada con control mecánico de malezas con cortadora sin revolvimiento del suelo periódicamente en el espacio entre las hileras y el herbicida en la hilera de plantío para los dos tratamientos. La conducción del huerto fue hecho después de las recomendaciones técnicas del Grupo Paulista (1994) para abonar y controlar las plagas y enfermedades cuando necesario. 
Las evaluaciones fueron hechas en 2002 (desarrollo de las plantas, producción, raíces y propiedades del suelo) y 2003 (producción y calidad de los fruto). La producción fue valorada a través del control del número de fruto por planta. El desarrollo de las plantas fue valorado con las medidas de altura, diámetro de las copas y perímetro del tronco $5 \mathrm{~cm}$ encima de la línea del injerto en seis repeticiones, con dos plantas por parcela. En la cosecha, una muestra de 20 frutos fue retirada por parcela para las determinaciones del peso, el jugo de las frutas, contenido de Vitamina $\mathrm{C}$, sólidos solubles totales (SST) por refractómetro, acidez titulable (AT) y relación SST / AT (ratio).

La evaluación de las raíces fue lograda por el método de la trinchera al final de la estación de lluvia (marzo) para observar el sistema radicular después de su desarrollo máximo, con cuatro repeticiones (trincheras) por tratamiento. Cuatro meses antes de la apertura de las trincheras, el sitio fue tratado con herbicida post emergencia (glifosate), para eliminar las malezas y facilitar la evaluación de las raíces de los cítricos. Las trincheras se abrieron desde el tronco hasta la mitad del espacio de la plantación en la entrelínea $(3,75 \mathrm{~m})$ y en la hilera de la plantación $(2 \mathrm{~m})$, con la profundidad de $1.0 \mathrm{~m}$, las paredes de las trincheras y las raíces fueron expuestos en la longitud de $1 \mathrm{~cm}$, con la ayuda de un rollo y pintura látex blanca. Se fue colocando en el muro del perfil un marco de madera con una tela de hilos de nylon, formando cuadros de $25 \times 25 \mathrm{~cm}$. De cada cuadro fue tomada una imagen digital. La cantidad de raíces en estas imágenes fue cuantificada con el software SIARCS. Fue calculada también la profundidad y la distancia efectivas del sistema radicular, donde se distribuyen $80 \%$ de las raíces.
Las muestras del suelo para los análisis químicos y físicos fueron sacados en las trincheras, con cuatro repeticiones por tratamiento, en los siguientes puntos: A (entrelínea a $0,75 \mathrm{~m}$ del tronco), B (entrelínea a 1,80 $\mathrm{m}$ del tronco), $\mathrm{C}$ (entrelínea a $3,65 \mathrm{~m}$ del tronco), D (hilera de plantío a 1,00 del tronco m) y E (línea de plantío a 2,00 $\mathrm{m}$ del tronco). Para todos los puntos fueron hechas muestras en las profundidades de $0,0-0,10 ; 0,10-0,20 ; 0,20-0,40 ; 0,40-0,60 ; 0,60$ 0,$80 ; 0,80-1,00 \mathrm{~m}$. Para la evaluación de la densidad del suelo se utilizo el método del anillo volumétrico con anillos de $0,05 \mathrm{~m}$ de diámetro y $0,047 \mathrm{~m}$ de altura. Para las evaluaciones de la resistencia del suelo a la penetración, fue llevado a cabo utilizando penetrómetro de impacto en los puntos A, B y C ya descritos. El análisis químico evaluó: materia orgánica, $\mathrm{P}, \mathrm{K}, \mathrm{Ca}, \mathrm{Mg}$, el pH y Al (Pavan et al., 1992). Los datos obtenidos fueron sometidos al examen de varianzas, en diseño completamente aleatorio, utilizando la prueba $\mathrm{t}$ de Student $(\mathrm{P}<\mathrm{a}$ $0,5)$.

\section{Resultados y Discusión}

El desarrollo y la producción de naranjas no han sido afectados por el sistema de preparación del terreno para la plantación (Tabla 1). También en el noroeste del Paraná Auler et al. (2004; 2008) señalaran que el sistema para preparar el terreno en rayas fue equivalente a prepararse el suelo con el sistema convencional para la producción de naranjas 'Valencia' y 'Pera', pero cuando se compararon distintos porta injertos, la lima Rangpur condujo a una menor producción en el sistema de plantar en rayas que en el convencional. 
Tabla 1. Características de desarrollo de plantas, de producción e de calidad de los frutos de naranja Valencia con dos sistemas de plantío.

\begin{tabular}{lrr}
\hline & Convencional & Cero labranza \\
\hline Altura de la planta (m) & 4,48 & 4,85 n.s. \\
Diámetro de la copa (m) & 4,95 & 4,98 n.s. \\
Perímetro del tronco (cm) & 64,6 & 63,5 n.s. \\
Número de frutos por planta (2002) & 814,4 & 696,3 n.s. \\
Número de frutos por planta (2003) & 611,8 & 634,0 n.s. \\
Peso medio do fruto (g) & 186,49 & 135,12 n.s. \\
Rendimiento en suco (\%) & 56,86 & 56,63 n.s. \\
Vitamina C (mg/100mL) & 58,51 & 60,32 n.s. \\
Acidez titulable - AT (\% ácido cítrico) & 1,40 & 1,51 n.s. \\
Sólidos solubles totales - SST ( ${ }^{\circ}$ Brix) & 10,46 & 11,05 n.s. \\
Ratio (SST/AT) & 7,48 & 7,38 n.s. \\
\hline
\end{tabular}

$*_{\text {n.s. }}=$ no significativo (prueba $t$ de Student, $\mathrm{P}<0,5$ ).

En los diversos trabajos que se han realizado en Brasil para estudiar el comportamiento de los huertos cítricos ya establecidos con diferentes sistemas de manejo del suelo, los resultados han indicado que en general, los sistemas que combinan una buena posición por la producción de frutas a una mejor conservación de las propiedades del suelo son aquellos que hacen la protección del suelo entre las líneas con plantas de cobertura, espontáneamente o implantadas al mismo tiempo que se minimiza la competencia por el agua durante la temporada seca (NEVES; DECHEN, 2001; CARVALHO et al., 2002). En el caso de este trabajo, como los tratamientos obligaron diferentes condiciones del suelo sólo durante la implantación del huerto, los tratamientos no alcanzaron a afectar la producción de las plantas, que comenzó la producción unos tres años después de la plantación.

La calidad de las frutas (peso, rendimiento del jugo, sólidos solubles totales, acidez total y la proporción) (Tabla 1), no ha sido tampoco influenciada por los tratamientos. Estos resultados están de acuerdo con las observaciones hechas por Sentelhas (2005), de que las condiciones climáticas ejercen una influencia decisiva sobre la calidad de la fruta cítrica.

Para la cantidad de raíces (Tabla 2), tanto en la hilera de plantío, como en la entrelínea y en total, se señala que no existía ninguna diferencia significativa entre los tratamientos para todas las profundidades estudiadas. Eso muestra que el sistema radicular de los cítricos probablemente logró vencer las diferencias impuestas por el sistema de plantación sobre el terreno. Otros trabajos con sistemas de manejo del suelo en huertos ya implantados también fracasó en detectar diferencias para la cantidad de raíces, aunque en algunos casos el manejo del suelo durante la manutención del huerto tenga afectado la distribución de las raíces en profundidad (NEVES; DECHEN, 2001). 
Tabla 2. Área de raíces de patrón lima Rangpur bajo copa de naranja 'Valencia' en dos sistemas de plantío en cuatro profundidades, en la hilera de plantío, en la entrelínea y en el total general.

\begin{tabular}{ccr}
\hline Tratamiento & \multicolumn{2}{c}{ Cero labranza } \\
\hline Profundidad $(\mathrm{m})$ & Área de raíces $\left(\mathrm{cm}^{2}\right)$ en la hilera de plantío $(\mathrm{H})$ & $156,46 \mathrm{a}$ \\
$0,00-0,25$ & $130,07 \mathrm{a}^{*}$ & $35,12 \mathrm{a}$ \\
$0,25-0,50$ & $35,73 \mathrm{a}$ & $39,57 \mathrm{a}$ \\
$0,50-0,75$ & $47,89 \mathrm{a}$ & $34,23 \mathrm{a}$ \\
$0,75-1,00$ & $49,51 \mathrm{a}$ & $265,38 \mathrm{a}$ \\
Tot. Hilera (H) & $263,20 \mathrm{a}$ & $170,92 \mathrm{a}$ \\
& Área de raíces $\left(\mathrm{cm}^{2}\right)$ en la interlínea (IL) & $65,46 \mathrm{a}$ \\
$0,00-0,25$ & $181,64 \mathrm{a}$ & $106,38 \mathrm{a}$ \\
$0,25-0,50$ & $71,61 \mathrm{a}$ & $98,07 \mathrm{a}$ \\
$0,50-0,75$ & $107,61 \mathrm{a}$ & $440,83 \mathrm{a}$ \\
$0,75-1,00$ & $114,74 \mathrm{a}$ & $706,21 \mathrm{a}$ \\
Tot. Interlínea(IL) & $475,60 \mathrm{a}$ & \\
Tot. General (H+IL) & $738,80 \mathrm{a}$ & $55,41 \mathrm{a}$ \\
& Profundidad efectiva de raíces $(\mathrm{cm})$ & $77,17 \mathrm{a}$ \\
\hline Hilera & $63,50 \mathrm{a}$ & $156,13 \mathrm{a}$ \\
Interlénea & $79,08 \mathrm{a}$ & $305,50 \mathrm{a}$ \\
\hline
\end{tabular}

*Medias con la misma letra en la línea no presentan diferencias significativas (prueba t de Student, $\mathrm{P}<0,5$ ).

Para la profundidad efectiva del sistema radicular, que indica la profundidad de la distribución del $80 \%$ de las raíces de la planta, no hubo ninguna diferencia significativa entre los tratamientos, con 0,55 a 0,63 en la hilera de plantío y 0,77 a 0,79 en la interlínea (Tabla 2). Estos resultados están de acuerdo con los obtenidos por Neves et al. (2004) y Bordin et al. (2005) en suelo arcilloso del norte del Paraná, pero son mayores que los de Vieira y Gomes (1999) que encontraron $0,50 \mathrm{~m}$ en la plantación de regadío y de Machado y Coelho (2000), que observaron 0,40 $\mathrm{m}$ en condiciones de no regadío, en Piracicaba, SP.

Fue señalado que llevando en consideración el tamaño de las trincheras ( $2 \mathrm{~m}$ en la hilera de plantación y 3,75 $\mathrm{m}$ en interlínea), en la hilera de plantío del sistema radicular es más superficial, y en la interlínea las raíces fueron mejor distribuidas en perfil del suelo. Esto puede atribuirse a un ambiente más favorable para el desarrollo de las raíces ofrecido por la mayor humedad del suelo bajo la copa de los árboles y la ausencia de otros tipos de vegetación en la hilera. La densa capa formada entre dos plantas en la línea de plantación, por el menor espacio en relación a interlínea, reduce la pérdida de agua del suelo por evaporación, formando un entorno favorable para el desarrollo, conduciendo a un aumento de la densidad de las raíces (CASTLE, 1980). Ya en las capas más profundas del suelo $(0,50-0,75$ y $0,75-1,00 \mathrm{~m})$ la mayor concentración de las raíces se notó en la interlínea, posiblemente, por la adaptación de las plantas en capturar el agua almacenada a mayor profundidad, evitando la competencia con las plantas de la cubierta de la vegetación en las interlíneas.

La distancia efectiva de las raíces fue de 1,56$1,57 \mathrm{~m}$ en la hilera de plantío y 2,96-3,05 m en la interlínea (Tabla 2), sin diferencia significativa entre los tratamientos. En el norte del Paraná en latossolo rojo muy arcilloso, Bordin et al. (2005) obtuvieron la distancia efectiva de $0,96-0,97$ en la hilera y $1,97-1,98 \mathrm{~m}$ en la interlínea. 
La distribución de las raíces horizontalmente presentó una relativamente uniformidad, tanto para la hilera de plantación como para la entrelínea, sin diferencias entre los dos sistemas de plantación (Figura 1). Esos resultados son diferentes de los de
Neves et al. (1998) que han encontrado marcada reducción en la cantidad de raíces a partir de 1,0 $\mathrm{m}$ del tronco para todos los sistemas de manejo evaluados, incluidos los sistemas de vegetación permanente en las interlíneas.

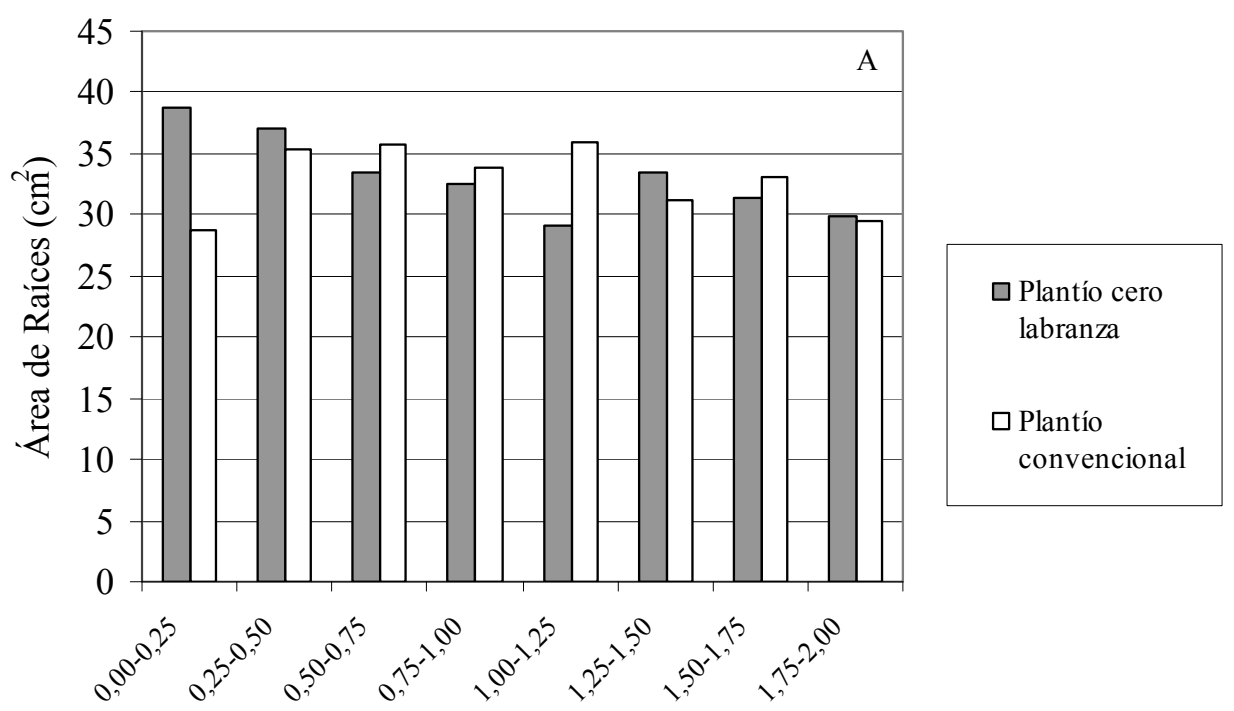

Distância del tronco (m)

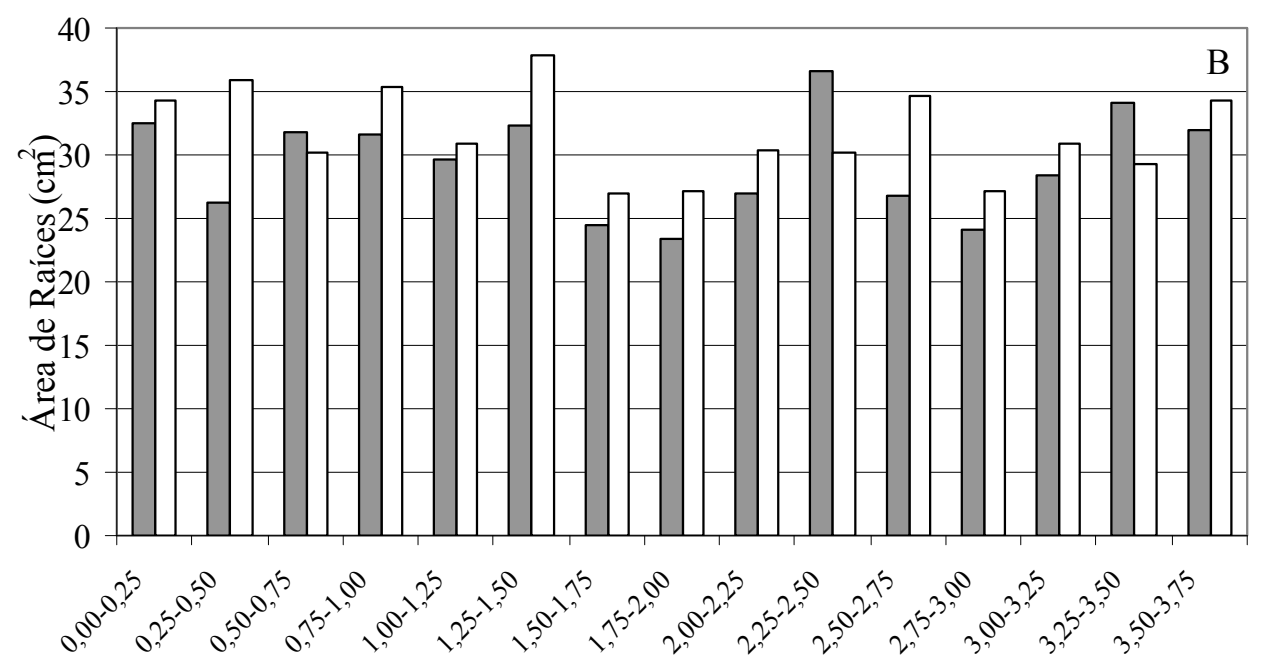

Distância del tronco (m)

Figura 1. Distribución de raíces de patrón lima Rangpur bajo copa de naranja 'Valencia', por distancia del tronco, en sistema de plantío directo y plantío convencional, en la hilera de plantío (A) y en la interlínea (B). 
El análisis químico del suelo mostró predominio del $\mathrm{pH}$ superior en el sistema de plantío convencional, principalmente para el muestreo realizado en las interlíneas en casi todas las profundidades evaluadas. Para las muestras de suelo en la línea de plantación hasta la profundidad de 0,20-0,40 m, no hay diferencias entre el $\mathrm{pH}$ del suelo de los dos sistemas de plantación. En este caso, al punto E, más distantes del tronco, para ninguno de las profundidades fue detectado diferencia de $\mathrm{pH}$ entre los tratamientos (Tabla 3). Eso puede estar relacionado con la forma de la incorporación de calcáreo en la plantación, que el sistema convencional se llevó a cabo por medio de la grada de discos seguida de labranza con arado cincel, mientras que en el sistema cero labranza se hizo sólo por apertura de surcos. En el punto B (a 1,80 m del tronco) el pH del suelo en el sistema convencional fue superior en la capa 0,40 0,60 m y punto en C (a 3,65 m del tronco) el sistema convencional tenían mayor $\mathrm{pH}$ en las capas $0-0,10 \mathrm{y}$ 0,20-0,40 cm, probablemente por su incorporación enl área total en el sistema convencional.

Para los niveles de $\mathrm{Ca}$ y $\mathrm{Mg}$ del suelo no se encontraron diferencias entre los sistemas de plantación para los diferentes puntos y profundidades evaluados. Sólo en la capa superficial del punto $\mathrm{C}$ (a 3,65 $\mathrm{m}$ del tronco), fue encontrado diferencia significativa entre los sistemas de plantación para el calcio. Este resultado puede atribuirse a una posible irregularidad en la distribución y la homogeneización del calcáreo. Generalmente, independientemente de la profundidad de y considerado, los niveles de $\mathrm{Ca}$ y $\mathrm{Mg}$ del suelo pueden clasificarse como bajos en conformidad con trabajos específicos para la cultura de cítricos (BAUMGARTNER; CABRITA, 2001; FIDALSKI; AULER, 1997). Para el aluminio no hubo diferencias entre los tratamientos (Tabla 3) y los niveles son bajos, característica del suelo de la región (FIDALSKI, 1997).

El contenido de materia orgánica del suelo (MOS) en general no ha sido afectado por el sistema de plantación (Tabla 3). Los niveles de MOS encontrados en este trabajo son superiores a los observados por Fidalski y Auler (1997) en huertos de la misma región, que encontraron que durante la fase de implantación del huerto en sistema convencional el MOS se redujo del nivel inicial de aproximadamente 5, $5 \mathrm{~g} \mathrm{dm}^{-3}$ (desde el original pastizales) para aproximadamente $3,5 \mathrm{~g} \mathrm{dm}^{-3}$ en la ocasión de la plantación en razón del revolvimiento y la exposición de la tierra durante su preparado, equilibrándose nuevamente durante los primeros años de la formación del huerto, alcanzando $7 \mathrm{~g} \mathrm{dm}^{-3}$ con siete anos de edad. En este trabajo, el contenido de MOS ya estaba en equilibrio y las posibles diferencias ocasionadas por nivelar el terreno en total del área en ocasión de la plantación no fueran siendo observadas.

Para el contenido de fósforo del suelo (Tabla 3), aunque se han detectado algunas diferencias entre tratamientos, con valores mayores en el área plantada en sistema convencional probablemente en razón de la incorporación de fertilizantes durante la plantación y de la reducida movilidad de este nutriente en el suelo, señalase que todas las muestras se consideraron con muy bajos niveles (GRUPO PAULISTA, 1994; BAUMGARTNER; CABRITA, 2001). Estos resultados están de acuerdo con la encuesta realizada por Fidalski (1997) en la misma región, que encontró que esta es la principal limitación de nutrición en el suelo del Noroeste de Paraná. Para el potasio, que generalmente presento niveles inferiores a los considerados apropiados para los huertos del estado de Sao Paulo (BAUMGARTNER; CABRITA, 2001), se observaron cambios significativos en las hileras de plantación, con más altos valores observados en las capas superficiales del suelo en el sistema convencional de la plantación, para los puntos D y E próximos a el tronco de las plantas (Tabla 3).

Para las condiciones físicas de los suelos, fueron observadas significativas diferencias en algunos puntos de muestreo y de profundidades. Para la densidad del suelo (Tabla 4) en el punto A (interlínea a $0,75 \mathrm{~m}$ del tronco) en la capa de 0, 20-0,40 m y punto E (hilera de plantío a 2,00m 
Tabla 3. Características químicas de un latossolo rojo distrófico bajo pomar de naranja 'Valência' sobre lima Rangpur, en dos sistemas de plantío en diferentes profundidades e puntos de coleta.

\begin{tabular}{|c|c|c|c|c|c|c|c|c|c|c|}
\hline \multirow[b]{3}{*}{ Prof. } & \multicolumn{10}{|c|}{ Punto de amostragem } \\
\hline & \multicolumn{2}{|c|}{ A (interlínea $0,75 \mathrm{~m}$ ) } & \multicolumn{2}{|c|}{ B (interlínea 1,80m) } & \multicolumn{2}{|c|}{ C(interlínea 3,65m) } & \multicolumn{2}{|c|}{ D (hilera 1,00m) } & \multicolumn{2}{|c|}{$\mathrm{E}$ (hilera 2,00m) } \\
\hline & Convenc. & $\begin{array}{c}\text { Cero } \\
\text { labranza }\end{array}$ & Convenc. & $\begin{array}{c}\text { Cero } \\
\text { labranza }\end{array}$ & Convenc. & $\begin{array}{c}\text { Cero } \\
\text { labranza }\end{array}$ & Convenc. & $\begin{array}{c}\text { Cero } \\
\text { labranza }\end{array}$ & Convenc. & $\begin{array}{c}\text { Cero } \\
\text { labranza }\end{array}$ \\
\hline$(\mathrm{cm})$ & \multicolumn{10}{|c|}{$\mathrm{pH}\left(\mathrm{CaCl}_{2}\right)$} \\
\hline $0-10$ & $5,29 \mathrm{a}^{*}$ & $4,38 \mathrm{~b}$ & $4,47 \mathrm{a}$ & $3,87 \mathrm{a}$ & $5,64 \mathrm{a}$ & $5,18 \mathrm{~b}$ & $5,36 \mathrm{a}$ & $4,58 \mathrm{a}$ & $5,24 \mathrm{a}$ & $4,78 \mathrm{a}$ \\
\hline $10-20$ & $3,24 \mathrm{a}$ & $2,89 \mathrm{a}$ & $2,51 \mathrm{a}$ & $2,55 \mathrm{a}$ & $4,37 \mathrm{a}$ & $4,56 \mathrm{a}$ & $3,94 \mathrm{a}$ & $3,51 \mathrm{a}$ & $4,20 \mathrm{a}$ & $3,91 \mathrm{a}$ \\
\hline $20-40$ & $3,73 \mathrm{a}$ & $2,85 \mathrm{~b}$ & $2,50 \mathrm{a}$ & $2,55 \mathrm{a}$ & $4,96 \mathrm{a}$ & $4,39 \mathrm{~b}$ & $4,09 \mathrm{a}$ & $3,20 \mathrm{~b}$ & $4,12 \mathrm{a}$ & $3,68 \mathrm{a}$ \\
\hline \multirow[t]{2}{*}{$40-60$} & $4,27 \mathrm{a}$ & $3,28 \mathrm{~b}$ & $3,39 \mathrm{a}$ & $2,94 \mathrm{~b}$ & $4,75 \mathrm{a}$ & $4,14 \mathrm{a}$ & $4,06 \mathrm{a}$ & $3,59 \mathrm{~b}$ & $3,99 \mathrm{a}$ & $3,79 \mathrm{a}$ \\
\hline & \multicolumn{10}{|c|}{ M.O. $\left(\mathrm{g} \mathrm{dm}^{-3}\right)$} \\
\hline $0-10$ & $14,85 \mathrm{a}$ & $12,48 \mathrm{a}$ & $11,45 \mathrm{a}$ & $10,80 \mathrm{a}$ & $11,80 \mathrm{a}$ & $14,18 \mathrm{a}$ & $12,45 \mathrm{a}$ & $13,50 \mathrm{a}$ & $13,17 \mathrm{a}$ & $14,72 \mathrm{a}$ \\
\hline $10-20$ & $10,80 \mathrm{a}$ & $9,10 \mathrm{a}$ & $14,80 \mathrm{a}$ & $8,07 \mathrm{~b}$ & $10,13 \mathrm{a}$ & $11,78 \mathrm{a}$ & $13,50 \mathrm{a}$ & $12,82 \mathrm{a}$ & $13,70 \mathrm{a}$ & $12,47 \mathrm{a}$ \\
\hline $20-40$ & $11,75 \mathrm{a}$ & $10,38 \mathrm{a}$ & $11,70 \mathrm{a}$ & $9,32 \mathrm{~b}$ & $10,03 \mathrm{a}$ & $12,03 \mathrm{a}$ & $8,70 \mathrm{~b}$ & $12,02 \mathrm{a}$ & $11,40 \mathrm{a}$ & $11,35 \mathrm{a}$ \\
\hline \multirow[t]{2}{*}{$40-60$} & $8,00 \mathrm{a}$ & $8,00 \mathrm{a}$ & $7,68 \mathrm{a}$ & $7,65 \mathrm{a}$ & $9,33 \mathrm{a}$ & $8,35 \mathrm{a}$ & $10,35 \mathrm{a}$ & $8,35 \mathrm{a}$ & $9,32 \mathrm{a}$ & $8,35 \mathrm{a}$ \\
\hline & \multicolumn{10}{|c|}{$\mathrm{P}\left(\mathrm{mg} \mathrm{dm}^{-3}\right)$} \\
\hline $0-10$ & $2,92 \mathrm{a}$ & $0,06 \mathrm{~b}$ & $7,77 \mathrm{a}$ & $0,21 \mathrm{~b}$ & $0,44 \mathrm{a}$ & $0,07 \mathrm{~b}$ & $0,53 \mathrm{a}$ & $0,07 \mathrm{a}$ & $1,43 \mathrm{a}$ & $0,06 \mathrm{a}$ \\
\hline $10-20$ & $7,53 \mathrm{a}$ & $0,54 \mathrm{~b}$ & $0,54 \mathrm{a}$ & $0,07 \mathrm{~b}$ & $0,39 \mathrm{a}$ & $0,14 \mathrm{a}$ & $0,38 \mathrm{a}$ & $0,07 \mathrm{~b}$ & $1,51 \mathrm{a}$ & $0,07 \mathrm{a}$ \\
\hline $20-40$ & $0,66 \mathrm{a}$ & $0,07 \mathrm{~b}$ & $0,54 \mathrm{a}$ & $0,07 \mathrm{~b}$ & $0,37 \mathrm{a}$ & $0,08 \mathrm{~b}$ & $3,55 \mathrm{a}$ & $0,06 \mathrm{a}$ & $4,84 \mathrm{a}$ & $0,66 \mathrm{a}$ \\
\hline $40-60$ & $0,56 \mathrm{a}$ & $0,08 \mathrm{~b}$ & $0,52 \mathrm{a}$ & $0,07 \mathrm{a}$ & $4,09 \mathrm{a}$ & $0,11 \mathrm{a}$ & $7,98 \mathrm{a}$ & $0,10 \mathrm{~b}$ & $0,40 \mathrm{a}$ & $0,07 \mathrm{~b}$ \\
\hline \multicolumn{11}{|c|}{$\mathrm{Al}\left(\mathrm{mmol}_{\mathrm{c}} \mathrm{dm}^{-3}\right)$} \\
\hline $0-10$ & $0,00 \mathrm{a}$ & $0,01 \mathrm{a}$ & $0,67 \mathrm{a}$ & $0,18 \mathrm{a}$ & $0,00 \mathrm{a}$ & $0,00 \mathrm{a}$ & $0,02 \mathrm{a}$ & $0,07 \mathrm{a}$ & $0,02 \mathrm{a}$ & $0,00 \mathrm{a}$ \\
\hline $10-20$ & $2,23 \mathrm{a}$ & $2,36 \mathrm{a}$ & $1,81 \mathrm{a}$ & $3,07 \mathrm{a}$ & $0,31 \mathrm{a}$ & $0,45 \mathrm{a}$ & $0,21 \mathrm{a}$ & $0,87 \mathrm{a}$ & $0,97 \mathrm{a}$ & $1,52 \mathrm{a}$ \\
\hline $20-40$ & $2,11 \mathrm{a}$ & $4,80 \mathrm{a}$ & $6,60 \mathrm{a}$ & $6,47 \mathrm{a}$ & $0,36 \mathrm{a}$ & $0,55 \mathrm{a}$ & $0,70 \mathrm{a}$ & $1,53 \mathrm{a}$ & $0,93 \mathrm{a}$ & $1,23 \mathrm{a}$ \\
\hline $40-60$ & $0,35 \mathrm{a}$ & $3,60 \mathrm{a}$ & $5,30 \mathrm{a}$ & $5,63 \mathrm{a}$ & $0,00 \mathrm{a}$ & $1,75 \mathrm{a}$ & $0,00 \mathrm{a}$ & $3,22 \mathrm{a}$ & $0,00 \mathrm{a}$ & $4,25 \mathrm{a}$ \\
\hline \multicolumn{11}{|c|}{$\mathrm{Ca}^{+}\left(\mathrm{mmol}_{\mathrm{c}} \mathrm{dm}^{-3}\right)$} \\
\hline $0-10$ & $8,0 \mathrm{a}^{*}$ & $6,7 \mathrm{a}$ & $5,2 \mathrm{a}$ & $3,7 \mathrm{a}$ & $8,2 \mathrm{a}$ & $6,2 \mathrm{~b}$ & $7,5 \mathrm{a}$ & $6,5 \mathrm{a}$ & $7,7 \mathrm{a}$ & $7,5 \mathrm{a}$ \\
\hline $10-20$ & $3,5 \mathrm{a}$ & $3,0 \mathrm{a}$ & $2,7 \mathrm{a}$ & $2,5 \mathrm{a}$ & $5,7 \mathrm{a}$ & $5,2 \mathrm{a}$ & $5,0 \mathrm{a}$ & $3,5 \mathrm{a}$ & $4,2 \mathrm{a}$ & $5,0 \mathrm{a}$ \\
\hline $20-40$ & $4,0 \mathrm{a}$ & $2,7 \mathrm{a}$ & $2,0 \mathrm{a}$ & $0,2,5 \mathrm{a}$ & $0,3,7 \mathrm{a}$ & $0,4,2 \mathrm{a}$ & $0,4,0 \mathrm{a}$ & $0,2,5 \mathrm{a}$ & $0,4,0 \mathrm{a}$ & $0,3,5 \mathrm{a}$ \\
\hline $40-60$ & $3,2 \mathrm{a}$ & $1,5 \mathrm{a}$ & $0,2 \mathrm{a}$ & $0,1,0 \mathrm{a}$ & $0,2,7 \mathrm{a}$ & $0,3,2 \mathrm{a}$ & $0,2,5 \mathrm{a}$ & $0,2,2 \mathrm{a}$ & $0,1,7 \mathrm{a}$ & $0,2,2 \mathrm{a}$ \\
\hline \multicolumn{11}{|c|}{$\operatorname{Mg}\left(\mathrm{mmol}_{\mathrm{c}} \mathrm{dm}^{-3}\right)$} \\
\hline $0-10$ & $6,2 \mathrm{a}$ & $4,7 \mathrm{a}$ & $6,2 \mathrm{a}$ & $7,2 \mathrm{a}$ & $6,7 \mathrm{a}$ & $9,5 \mathrm{a}$ & $5,7 \mathrm{a}$ & $4,7 \mathrm{a}$ & $6,5 \mathrm{a}$ & $6,0 \mathrm{a}$ \\
\hline $10-20$ & $6,2 \mathrm{a}$ & $7,0 \mathrm{a}$ & $4,0 \mathrm{a}$ & $6,0 \mathrm{a}$ & $9,5 \mathrm{a}$ & $6,5 \mathrm{a}$ & $5,7 \mathrm{a}$ & $6,5 \mathrm{a}$ & $9,7 \mathrm{a}$ & $5,5 \mathrm{a}$ \\
\hline $20-40$ & $4,2 \mathrm{a}$ & $4,2 \mathrm{a}$ & $2,0 \mathrm{a}$ & $4,0 \mathrm{a}$ & $7,2 \mathrm{a}$ & $8,2 \mathrm{a}$ & $6,7 \mathrm{a}$ & $5,2 \mathrm{a}$ & $4,5 \mathrm{a}$ & $8,2 \mathrm{a}$ \\
\hline $40-60$ & $7,2 \mathrm{a}$ & $7,0 \mathrm{a}$ & $3,0 \mathrm{a}$ & $6,0 \mathrm{a}$ & $7,0 \mathrm{a}$ & $7,5 \mathrm{a}$ & $6,0 \mathrm{a}$ & $9,0 \mathrm{a}$ & $9,0 \mathrm{a}$ & $8,0 \mathrm{a}$ \\
\hline \multicolumn{11}{|c|}{$\mathrm{K}\left(\mathrm{mmol}_{\mathrm{c}} \mathrm{dm}^{-3}\right)$} \\
\hline $0-10$ & $0,51 \mathrm{a}$ & $0,32 \mathrm{a}$ & $1,98 \mathrm{a}$ & $0,57 \mathrm{a}$ & $0,51 \mathrm{a}$ & $0,38 \mathrm{a}$ & $0,44 \mathrm{a}$ & $0,19 \mathrm{~b}$ & $0,32 \mathrm{a}$ & $0,06 \mathrm{~b}$ \\
\hline $10-20$ & $0,70 \mathrm{a}$ & $0,64 \mathrm{a}$ & $0,57 \mathrm{a}$ & $0,32 \mathrm{a}$ & $0,51 \mathrm{a}$ & $0,32 \mathrm{a}$ & $0,44 \mathrm{a}$ & $0,19 \mathrm{~b}$ & $0,38 \mathrm{a}$ & $0,25 \mathrm{a}$ \\
\hline $20-40$ & $0,44 \mathrm{a}$ & $0,32 \mathrm{a}$ & $0,64 \mathrm{a}$ & $0,25 \mathrm{a}$ & $0,38 \mathrm{a}$ & $0,19 \mathrm{a}$ & $0,51 \mathrm{a}$ & $0,19 \mathrm{a}$ & $0,76 \mathrm{a}$ & $0,96 \mathrm{a}$ \\
\hline $40-60$ & $0,38 \mathrm{a}$ & $0,32 \mathrm{a}$ & $0,51 \mathrm{a}$ & $0,19 \mathrm{~b}$ & $0,32 \mathrm{a}$ & $0,51 \mathrm{a}$ & $0,96 \mathrm{a}$ & $0,44 \mathrm{a}$ & $1,09 \mathrm{a}$ & $0,19 \mathrm{a}$ \\
\hline
\end{tabular}

$\mathrm{A}=$ interlínea a $0,75 \mathrm{~m}$ del tronco; $\mathrm{B}=$ interlínea a $1,80 \mathrm{~m}$ del tronco; $\mathrm{C}=$ interlínea a 3,65 $\mathrm{m}$ del tronco; $\mathrm{D}=$ hilera de plantío 1,00 $\mathrm{m}$ del tronco; $\mathrm{E}=$ hilera de plantío a $2,00 \mathrm{~m}$ del tronco.

*Medias con la misma letra en la línea, entre los sistemas de plantío, no presentan diferencias significativas (prueba t de Student, $\mathrm{P}<0,5)$. 
del tronco) en la capa $0,20-0,40 \mathrm{~m}$, la densidad fue mayor en el sistema de preparado del suelo cero labranza que en el convencional, mientras en todos los demás puntos y profundidades no se encontraron diferencias. Generalmente, el suelo tuvo altos valores de densidad, incluida la corona y la hilera de la plantación (A, D y E), donde el tránsito de maquinas es menor. Probablemente en la plantación, el sistema convencional debe haber contribuido a reducir la densidad de suelo; en tanto, tras 11 años de localizar el plantío del huerto, esta diferencia no se ha detectado. Cintra, Libardi y Jorge (1999) señalaron que en suelo arenoso del nordeste brasileño, el aumento en los valores de densidad de la tierra de 1,29-1,35 para 1,44 $\mathrm{g} \mathrm{cm}^{-3}$ obligaron a la reducción de la cantidad de raíces de los cítricos. Por otro lado, Neves et al. (2004) observaron un normal desarrollo de las plantas y de las raíces de lima Rangpur injertada con lima ácida Tahití en suelo muy arcilloso del norte del Paraná con densidades de hasta $1,68 \mathrm{~kg} \mathrm{dm}^{-3}$.

Para la resistencia mecánica a la penetración en el punto A (interlínea a 0,75 del tronco) no existío ninguna diferencia significativa entre los tratamientos, ya en el punto B (interlínea a 1, 80 $\mathrm{m}$ del tronco) y en el punto $\mathrm{C}$ (entrefina a $3,65 \mathrm{~m}$ del tronco) en profundidad superficial, el sistema de plantío convencional presento una mayor resistencia que el preparo con cero labranza (Tabla 4). Ese hecho puede estar relacionado con el reordenamiento de las partículas del suelo que se produce después de la movilización, especialmente si después del revolvimiento ocurre la lluvia fuerte, lo que aumentará el efecto de compactación del suelo.

Tabla 4. Densidad del suelo y resistencia à la penetración de un latossolo rojo distrófico bajo pomar de naranja 'Valência' sobre lima Rangpur, en dos sistemas de plantío, en diferentes profundidades y puntos de coleta.

\begin{tabular}{|c|c|c|c|c|c|c|c|c|c|c|}
\hline \multirow[b]{3}{*}{ Profund. } & \multicolumn{10}{|c|}{ Local de amostragem } \\
\hline & \multicolumn{2}{|c|}{ A (interlínea0,75m) } & \multicolumn{2}{|c|}{ B (interlínea1,80m) } & \multicolumn{2}{|c|}{ C (interlínea3,65m) } & \multicolumn{2}{|c|}{$\mathrm{D}$ (hilera $1,00 \mathrm{~m}$ ) } & \multicolumn{2}{|c|}{ E (hilera $2,00 \mathrm{~m}$ ) } \\
\hline & Convenc. & $\begin{array}{c}\text { Cero } \\
\text { labranza }\end{array}$ & Convenc. & $\begin{array}{c}\text { Cero } \\
\text { labranza }\end{array}$ & Convenc. & $\begin{array}{c}\text { Cero } \\
\text { labranza }\end{array}$ & Convenc. & $\begin{array}{c}\text { Cero } \\
\text { labranza }\end{array}$ & Convenc. & $\begin{array}{c}\text { Cero } \\
\text { labranza }\end{array}$ \\
\hline$(\mathrm{cm})$ & \multicolumn{10}{|c|}{ Densidad del solo $\left(\mathrm{g} \mathrm{dm}^{-3}\right)$} \\
\hline $0-10$ & $1,76 \mathrm{a} *$ & $1,80 \mathrm{a}$ & $1,78 \mathrm{a}$ & $1,83 \mathrm{a}$ & $1,81 \mathrm{a}$ & $1,93 \mathrm{a}$ & $1,72 \mathrm{a}$ & $1,80 \mathrm{a}$ & $1,72 \mathrm{a}$ & $1,74 \mathrm{a}$ \\
\hline $10-20$ & $1,80 \mathrm{a}$ & $1,92 \mathrm{a}$ & $1,77 \mathrm{a}$ & $1,86 \mathrm{a}$ & $1,79 \mathrm{a}$ & $1,88 \mathrm{a}$ & $1,84 \mathrm{a}$ & $1,93 \mathrm{a}$ & $1,74 \mathrm{a}$ & $1,87 \mathrm{~b}$ \\
\hline $20-40$ & $1,76 \mathrm{a}$ & $1,97 \mathrm{~b}$ & $1,86 \mathrm{a}$ & $1,81 \mathrm{a}$ & $1,87 \mathrm{a}$ & $1,88 \mathrm{a}$ & $1,83 \mathrm{a}$ & $1,91 \mathrm{a}$ & $1,74 \mathrm{a}$ & $1,94 \mathrm{~b}$ \\
\hline $40-60$ & $1,83 \mathrm{a}$ & $1,88 \mathrm{a}$ & $1,73 \mathrm{a}$ & $1,80 \mathrm{a}$ & $1,76 \mathrm{a}$ & $1,90 \mathrm{a}$ & $1,79 \mathrm{a}$ & $1,90 \mathrm{a}$ & $1,81 \mathrm{a}$ & $1,88 \mathrm{a}$ \\
\hline & \multicolumn{10}{|c|}{ Resistencia à la penetración (Mpa) } \\
\hline $0-10$ & $2,38 \mathrm{a}$ & $2,15 \mathrm{a}$ & $3,74 \mathrm{~b}$ & $1,80 \mathrm{a}$ & a $\quad 6,02 b$ & $1,92 \mathrm{a}$ & - & - & - & - \\
\hline $10-20$ & $6,25 \mathrm{a}$ & $4,47 \mathrm{a}$ & $5,78 \mathrm{a}$ & $2,85 \mathrm{a}$ & a $\quad 4,59$ a & $4,36 \mathrm{a}$ & - & - & - & - \\
\hline $20-30$ & $6,33 \mathrm{a}$ & $13,51 \mathrm{a}$ & $5,40 \mathrm{a}$ & 4,93 a & a $\quad 4,27 \mathrm{a}$ & $4,70 \mathrm{a}$ & - & - & - & - \\
\hline $30-40$ & $7,07 \mathrm{a}$ & $9,80 \mathrm{a}$ & $7,10 \mathrm{a}$ & $3,77 \mathrm{a}$ & a $\quad 3,81 \mathrm{a}$ & $4,36 \mathrm{a}$ & - & - & - & - \\
\hline $40-50$ & $8,09 \mathrm{a}$ & $5,71 \mathrm{a}$ & $5,86 \mathrm{a}$ & $4,24 \mathrm{a}$ & a $\quad 3,66 \mathrm{a}$ & $6,32 \mathrm{~b}$ & - & - & - & - \\
\hline
\end{tabular}

$\mathrm{A}=$ interlínea a $0,75 \mathrm{~m}$ del tronco; $\mathrm{B}=$ interlínea a $1,80 \mathrm{~m}$ del tronco; $\mathrm{C}=$ interlínea a $3,65 \mathrm{~m}$ del tronco; $\mathrm{D}=$ hilera de plantío 1,00 $\mathrm{m}$ del tronco; $\mathrm{E}=$ hilera de plantío a $2,00 \mathrm{~m}$ del tronco.

*Medias con la misma letra en la línea, entre los sistemas de plantío, no presentan diferencias significativas (prueba t de Student, $\mathrm{P}<0,5)$. 


\section{Conclusiones}

La producción no es afectada por los tratamientos. Los sistemas de plantación tampoco afectan el desarrollo de las plantas y de las raíces y la calidad de los frutos. En el sistema de plantación convencional, el suelo presenta mayor resistencia a la penetración en la interlínea, que en el sistema de plantación con cero labranza.

\section{Agradecimientos}

Los autores agradecem a Atílio Tormem por permitir la realización de los trabajos en su huerto e a el Conselho Nacional de Desenvolvimento Científico e Tecnológico por el suporte financiero.

\section{Referencias}

AULER, P. A. M.; FIDALSKI, J.; PAVAN, M. A..; GOMES, J. C.; JACOMINO, A. P. Sistema de preparo do solo, calagem e porta-enxertos para a produção de laranja Valência na região noroeste do Paraná. In: CONGRESSO BRASILEIRO DE FRUTICULTURA, 18., Florianópolis, 2004. Resumos... Florianópolis: SBF, 2004. CD-ROM.

AULER, P. A. M.; FIDALSKI, J.; PAVAN, M. A.; NEVES, C. S. V. J. Produção de laranja 'Pêra' em sistemas de preparo de solo e manejo nas entrelinhas. Revista Brasileira de Ciência do Solo, Viçosa, MG, v. 32, n. 1, p. 363-374, 2008.

BAUMGARTNER, J. G.; CABRITA, J. R. M. Avaliação nutricional de pomares cítricos na região de Bebedouro. Jaboticabal: Funep, 2001, 24 p.

BORDIN, I.; NEVES, C. S. V. J.; AIDA, F. T.; SOUZA, W. R.; DAVOGLIO JÚNIOR, A. C.; FURLANETO, T. L. R.; TAVARES FILHO, J. Sistema radicular de plantas cítricas e atributos físicos do solo num latossolo argiloso submetido à escarificação. Ciência Rural, Santa Maria, v. 35, n. 4, p. 820-825, 2005.

CARVALHO, J. E. B.; SOUZA, L. S. C.; RANULFO, C.; ANTAS, P. E. U. T.; ARAÚJO, A. M. A.; LOPES, L. C.; SANTOS, R. C.; LOPES, N. C. M.; SOUZA, A. L. $\mathrm{V}$. Leguminosa no controle integrado de plantas daninhas para aumentar a produtividade da laranja-'Pêra'. Revista Brasileira de Fruticultura, Jaboticabal, v. 24, n. 1, p. 8285, 2002.
CASTLE, W. S. Fibrous root distribution of 'Pinaple' Orange trees on Rough Lemon rootstock at three tree spacings. Journal of the American Society for Horticultural Science, Alexandria, v. 105, n. 3, p. 478480, 1980.

CINTRA, F. L. D.; LIBARDI, P. L.; JORGE, L. A. C. Distribuição do sistema radicular de porta-enxertos de citros em ecossistema de tabuleiro costeiro. Revista Brasileira de Fruticultura, Jaboticabal, v. 21, n. 3, p. 313-317, 1999.

FIDALSKI, J. Fertilidade do solo sob pastagens, lavouras anuais e permanentes na região Noroeste do Paraná. Revista Unimar, Maringá, v. 19, n. 3, p. 853-861, 1997.

FIDALSKI, J.; AULER, P. A. M. Levantamento nutricional de pomares de laranja no noroeste do Paraná. Arquivos de Biologia e Tecnologia, Curitiba, v. 40, n. 2, p. 443-451, 1997.

GRUPO PAULISTA DE ADUBAÇÃO E CALAGEM PARA CITROS - GPACC. Recomendações de adubação e calagem para citros no estado de São Paulo. Laranja, Cordeirópolis, v. 15, p. 1-27, 1994. Edição Especial.

MACHADO, C. C.; COELHO, R. D. Estudo da distribuição espacial do sistema radicular do limão 'Cravo' enxertado com lima ácida 'Tahiti'. Laranja, Cordeirópolis, v. 21, p. 359-380, 2000.

MARTINS, A.; PINTO, G. Efeitos da ripagem localizada versus ripagem contínua nas propriedades do solo e na resposta das plantas em novos povoamentos florestais. Silva Lusitana, Lisboa, v. 12, n. 2, p. 191-202, 2004.

MUZILLI, O. Ocupação e uso do solo agrícola no Paraná. In: CASTRO FILHO, C.; MUZILLI, O. (Ed.). Uso e manejo dos solos de baixa aptidão. Londrina: IAPAR, 1999. p. 8-12 (Circular Técnica IAPAR, 108).

NEVES, C. S. V. J.; DECHEN, A. R.; FELLER, C.; ABI-SAAB, O. J. G.; PIEDADE, S. M. S. Efeito do manejo do solo no sistema radicular de tangerineira Poncã enxertada sobre limoeiro Cravo em latossolo roxo. Revista Brasileira de Fruticultura, Cruz das Almas, v. 20, n. 2, p. 246-253, 1998.

NEVES, C. S. V. J.; DECHEN, A. R. Sistemas de manejo de solo em pomar de tangerina Poncã sobre limão Cravo em latossolo roxo. Laranja, Cordeiróplis, v. 22, n. 1, p. 167-184, 2001.

NEVES, C. S. V. J.; MURATA, I. M.; STENZEL, N. M. C.; MEDINA, C. C.; BORGES, A. V.; OKUMOTO, S. H.; HIDEKI, S.; LEE, R. H. C.; KANAI, H. T. Root distribution of rootstocks for 'Tahiti' lime. Scientia Agricola, Piracicaba, v. 61, n. 1, p. 94-99, 2004. 
PAVAN, M. A.; BLOCH, M. F.; ZEMPULSKI, H. C.; MIYAZAWA, M.; ZOCOLER, D. C. Manual de análise química de solo e controle de qualidade. Londrina: Instituto Agronômico do Paraná, 1992. 38 p. (Circular IAPAR, 76)

RUFINO, R. L.; MUZILLI, O.; PAVAN, M. A. Manejo do solo. In: INSTITUTO AGRONÔMICO DO PARANÁ. $A$ Citricultura no Paraná. Londrina: IAPAR, 1992. p. 5390. (Circular, 72).
SENTELHAS, P. C. Agrometeorologia dos citros. In: MATTOS JUNIOR, D.; DE NEGRI, J. D.; PIO, R. M.; POMPEU JUNIOR, J. (Org.). Citros. Campinas: Instituto Agronômico e Fapesp, 2005. v. 1. p. 317-344.

VIEIRA, D. B.; GOMES, E. M. Determinação da profundidade efetiva do sistema radicular do limão 'Cravo' com copa de lima ácida 'Tahiti'. Laranja, Cordeirópolis, v. 20, p. 419-431, 1999. 
